\title{
A New Approach in the Study of Hypertension in the Elderly Subject: The Role of Epigenetics
}

\author{
Valerio Massimo Magro*1, Carla Coppola ${ }^{2}$, Michele Caturano ${ }^{3}$, Giovanni Scala ${ }^{4}$ and Walter Verrusio ${ }^{5}$ \\ ${ }^{1}$ Department of Internal Medicine and Geriatrics, Italy \\ ${ }^{2}$ Department of Intensive Rehabilitation, Italy \\ ${ }^{3}$ Department of Internal Medicine, Italy
}

${ }^{4}$ ASL RM2 Home Care Center, Rome

${ }^{5}$ Department of Cardiovascular, Italy

*Corresponding author: Valerio Massimo Magro, Department of Internal Medicine and Geriatry, Italy.

Received Date: January 18, 2019

Published Date: January 24, 2019

\section{Introduction}

The causes of high blood pressure are numerous and are based on the important distinction between the various types of arterial hypertension, which sees the existence of so-called essential hypertension and the so-called secondary hypertension. The elderly, considered as individuals 75 years of age and older, with essential hypertension, represent a conspicuous percentage of subjects suffering from diseases in the general population. Essential hypertension is the hypertensive state whose presence is imputed to a multiplicity of predisposing factors, since it is impossible to establish with certainty a precise and univocal triggering cause. Age itself is a powerful risk factor for hypertension. Although it has never been set aside, the emphasis has recently been placed on the role played by genetics in the genesis of hypertension. Furthermore, the discovery that changes in the functioning of a gene do not derive only from mutations in the nucleotide sequence but also that changes in the conformation of chromatin and modifications of the subsequent phases of gene expression, from the transcription of mRNA onwards, led to consider the role played by the genetic heritage in the determinism of this pathology under a new aspect.

\section{Discussion}

Epigenetics is considered to be that series of dynamic and reversible modifications, generated and removed by enzymes, of our genetic heritage. There are therefore changes in gene activity that do not involve changes in the DNA sequence and that are also inheritable. The structure of the chromosome is the nucleosome, that is a formation of structural proteins of the chromosome itself, the histones, which, in number of eight, form a complex. The DNA helix surrounds around the complex. In fact the chromatin is present in the cell nucleus in different degrees of compaction. There is a condensed chromatin (heterochromatin), which is transcriptionally silent, because the DNA is not accessible. The histone tails protrude from the nucleosome and they participate in the compaction of the chromatin. There are enzymes that modify histones or DNA itself through acetylation or methylation. For example, in case of histone acetylation, the nucleosome remodels, the chromatin is decondensed and the transcription is activated; another type of enzyme performs a deacetylation action on the tail of the histones: the chromatin is recondensed and the transcription is repressed.

In contrast, the DNA methylation is involved in the inactivation of many genes. The transcriptionally active chromatin is hyperacetylated and de methylated. How does all of this correlate with hypertension? For example, smooth muscle cells are the major cell type in blood vessels and their function is to regulate blood flow and pressure through vessel wall contraction and relaxation. The contractile phenotype of these cells are characterized by a repertoire of contractile proteins (smooth muscle alpha actin, ACTA2; smoothelin; h-caldesmon; calponin; transgelin, TALGN; smooth muscle myosin heavy chain, MYH11). Many of the genes that characterize the phenotypic type of these cells, including the contractile proteins, are regulated at the level of transcription. There is an evidence of a role of epigenetic modification, particularly histone modification, in controlling gene expression during normal smooth muscle cells differentiation and function and phenotypic modulation. A review of 2015 summarize this epigenetic regulation [1]. 
The role of diet and take the salt in the genesis of hypertension is still a topic widely discussed. The progression of salt-sensitive hypertension and renal disease is dramatically altered in salt sensitive rats fed diets containing different sources of protein. These mice are characterized by having a different chromosomal arrangement compared to other mice and The magnitude of transcriptome difference between the normal rat and the salt sentive rat correlates with the magnitude of genetic difference to some extent but also with phenotypic differences [2]. A recent study for example showed that um specific enzyme that is known as being associated with the salt sensitivity of hypertension, called lysine specific demethylase 1 (LSD1), has a gene with an expression that is was modified by dietary salt; the LSD1+/-mouse may serve as a model for salt-sensitivity in human with essential hypertension [3]. Together with the exposed study there are several data that indicate the existence of an extensive convergence and divergence of genomic effects that cannot be explained solely by the genomic sequence but there is also an epigenetic regulatory mechanism [4] Figure 1.

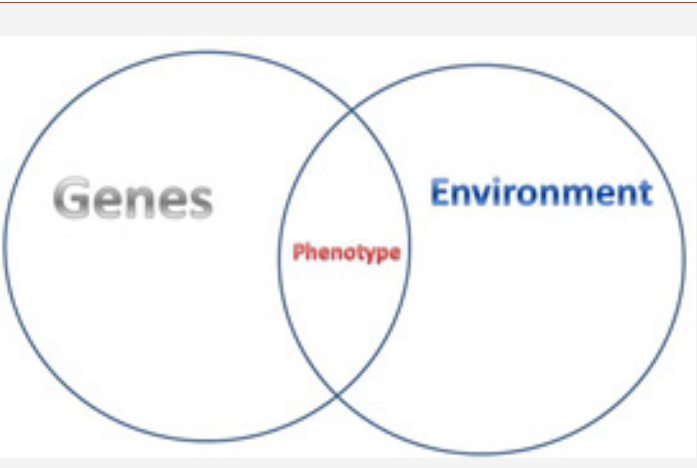

Figure 1: There are multiplicity of predisposing factors and conditions, in the hypertension, that depend on a complex but interactive genetic and environmental system to influence the final phenotype of the patients.

To give another example, using animal models, a study by Angrisano S et al. demonstrated that transcriptional reprogramming of Atp2a2 (encoding for sarcoplasmic reticulum Ca2+ ATPase, also responsible for Darier's disease) and Myh7 (encoding for betamyosin-heavy chain) genes, whose expression levels are considered a hallmark of essential hypertension, in the mouse model are associated with significant changes in the methylation status of chromatin, modifying its dynamics at the promoter regions of these genes. The myosin heavy-chain-associated RNA transcript was also found downregulated in conditions of pressure overload resulting in essential hypertension: antagonizing the function of Brg1, a stress-activated ATP-dependent chromatin-remodelling factor implicated in the regulation of pathological gene expression myosin heavy-chain-associated RNA transcript interferes with its binding to the DNA targets, preventing the expression of stress-induced genes $[5,6]$. DNA methylation and histone modification involving genes that affect the renin angiotensin aldosterone system, ionic and renal sodium balance is equally important and with clinical relapses: in fact the modification of renin-angiotensin-aldosterone system, a hormone system that is integral to the physiological regulation of blood pressure, is important both in the genesis of hypertension and also constitutes a possible therapeutic target.
Thus, modifications of methylation, both in hypo and hyper sense, of genes such as angiotensin-converting enzyme 1 (ACE1), hydroxysteroid dehydrogenase-11_2 enzyme (HSD11B2, responsible for degrading cortisol) and Sslc12a2 (gene that codes for the protein $\mathrm{Cl}$ - coupled cation cotransportes or $\mathrm{NKCC} 1, \mathrm{Na}^{+}$ $, \mathrm{K}^{+}, 2 \mathrm{Cl}^{-}$cotransporters) have been observed to be implicated in hypertension both in the guinea pig and in the human subject. Equally, the hypomethylation of the promoter regions of the angiotensin 2 type 1 receptor gene, in the adrenal glands of the maternal low protein rat exhibited hypertension in response to salt intake [7-10]. Furthermore, the enzyme endothelial nitric oxide synthase (eNOS) is primarily responsible for the production of nitric oxide in the vascular endothelium, and plays a key role in the regulation of vascular tone; eNOS also appears involved in the processes that regulate aging - for example, in a work of Nisoli E et al, the Authors observed the possible involvement of eNOS, given that the eNOS mice lacking the enzyme (and therefore deficient in the genesis of mitochondria) showed a minor survival induced by aging [9] - and a (positive) impact in some chronic diseases of the elderly. eNOS expression appears to be controlled by cell-specific histone modifications $[10,11]$. Then there is the role played by genetic material that regulates other genetic material. Non-coding RNA in molecular biology is a gene transcript that does not go into traspription; this means that the gene coding for that RNA does not code for a protein but simply for an RNA molecule (and therefore not an mRNA, because otherwise it would go into trascription).

So, non-coding RNA can perform different functions including that of regulating other transcripts. Always in animal models, there are studies In this study to identify long non-coding RNAs in the rat genome and particularly at the level of the smooth muscle cells in blood vessels, to demonstrate their role in the response of the smooth muscle cells to the angiotensin 2 and therefore the role of misregulation of angiotensin 2 in the pathogenesis of hypertension, too [12]. Other smaller RNAs, known as microRNAs (miRNAs), seem to play some roles in almost all biological processes; their function predominantly consist as sequence-targeted modifiers of gene expression through inhibition of post-transcriptional processes. The miRNAs have been found to be associated with specific cardiovascular diseases, yet, including hypertension: particularly, miRNAs associated with hypertension are miR-143/-145, whose targets are angiotensin converting enzyme (ACE) and KLF4/5, which are repressors in smooth muscle cells phenotype-switching processes [13]. Many miRNAs appear to be (dys)regulated during cellular senescence and ageing, so that the study of miRNAs in the elderly patients suffering from high blood pressure may be of interest $[14,15]$.

\section{Conclusion}

The arterial hypertension perhaps is the prototype of pathologies in which the interaction between genes and the environment plays a major role in the genesis and maintenance of the high blood pressure values. At the same time, we are more and more delineating the interactive mechanisms in which the external environment is acting on the genome, modifying it, with consequent aging of the apparatuses and the onset of pathologies that are undermining the health of the organism [16]. Nothing 
prevents us from believing that the pathways of research, clinical and eminently gerontological, cannot overlap. Certainly greater attention to genetic factors and the role of epigenetics in arterial hypertension will be the focus of new research in the field of hypertension in the elderly (and not).

\section{Acknowledgement}

None.

\section{Conflict of Interest}

No conflict of interest.

\section{References}

1. Liu R, Leslie KL, Martin KA (2015) Epigenetic regulation of smooth muscle cell plasticity. Biochim Biophys Acta 1849(4): 448-453.

2. Mori T, Polichnowski A, Glocka P, Kaldunski M, Ohsaki Y, et al. (2008) High Perfusion Pressure Accelerates Renal Injury in Salt-Sensitive Hypertension. J Am Soc Nephrol 19(8): 1472-1482.

3. Williams JS, Chamarthi B, Goodarzi MO, Pojoga LH, Sun B, et al. Lysinespecific demethylase 1: an epigenetic regulator of salt-sensitive hypertension. Am J Hypertens 25(7): 812-817.

4. Liang M, Cowley AW Jr, Mattson DL, Kotchen TA, et al. (2013) Epigenomics of hypertension. Semin Nephrol 33(4): 392-399.

5. Angrisano T, Schiattarella GG, Keller S, Pironti G, Florio E, et al. (2014) Epigenetic switch at atp2a2 and myh7 gene promoters in pressure overloadinduced heart failure. PLoS One 9 (9): e106024.

6. De Majo F, Calore M (2018) Chromatin remodelling and epigenetic state regulation by non-coding RNAs in the diseased heart. Noncoding RNA Res 3(1): 20-28.
7. Orlov SN, Koltsova SV, Kapilevich LV, Svetlana V Gusakova, Nickolai O Dulin, et al. (2015) NKCC1 and NKCC2: The pathogenetic role of cationchloride cotransporters in hypertension. Genes Dis 2(2): 186-196.

8. Stoll S, Wang C, Qiu H (2018) DNA Methylation and Histone Modification in Hypertension. Int J Mol Sci 19(4): 1174.

9. Valerio A, Nisoli E (2015) Nitric oxide, interorganelle communication, and energy flow: a novel route to slow aging. Front Cell Dev Biol 3: 6 .

10. Wise IA, Charchar FJ (2016) Epigenetic Modifications in Essential Hypertension. Int J Mol Sci 17(4): 451.

11. Wood KC, Cortese-Krott MM, Kovacic JC, Audrey Noguchi BS, Virginia B Liu, et al. (2013) Circulating Blood eNOS Contributes to the Regulation of Systemic Blood Pressure and Nitrite Homeostasis. Arterioscler Thromb Vasc Biol 33(8): 10.

12. Leung A, Trac C, Jin W, Lanting L, Akbany A, et al. (2003) Novel long noncoding RNAs are regulated by angiotensin II in vascular smooth muscle cells. Circ Res 113(3): 266-278.

13. Maegd efessel L (2014) The emerging role of microRNAs in cardiovascular disease. J Intern Med. 276(6): 633-644.

14. Chen S, Chen R, Zang T, Lin S, Chen Z, et al. (2018) Relationship of cardiovascular disease risk factors and noncoding RNAs with hypertension: a case-control study. BMC Cardiovascular Disorders 18: 58.

15. Nanoudis S, Pikilidou M, Yavropoulou M, Zebekakis P, et al. (2017) The Role of MicroRNAs in Arterial Stiffness and Arterial Calcification. An Update and Review of the Literature. Front Genet 8: 209.

16. Dobrynina LA, Zabitova MR, Kalashnikova LA, Gnedovskaya EV, Piradov MA, et al. (2018) Hypertension and Cerebral Microangiopathy (Cerebral Small Vessel Disease): Genetic and Epigenetic Aspects of Their Relationship. Acta Naturae 10(2): 4-15. 